AIMS Biophysics, 8(1): 111-120.

http://www.aimspress.com/journal/biophysics

Research article

\title{
Charged amino acids may promote coronavirus SARS-CoV-2 fusion with the host cell
}

\section{Piotr H. Pawłowski*}

Institute of Biochemistry and Biophysics, Polish Academy of Sciences, Warszawa, Poland

* Correspondence: Email: piotrp@ibb.waw.pl; Tel: +48226597072.

\begin{abstract}
The charged amino acids in the spike protein of SARS-CoV-2 exhibit some specific distribution. In the RBD region of the $\mathrm{S} 1$ unit the positive charge dominates in the protein interior and the negative charge prevails on the surface exposed towards ACE2 receptor. The charged amino acids in the S2 region preceding heptad repeats of the spike protein of SARS-CoV-2, exhibit a central concentration, and the imbalance of a positive charge. The idea that both distributions of charged amino acids may, in an electrostatic manner, facilitate the coronavirus infection of the cell is presented. An evaluation of the virus-receptor binding energy, by docking the peptide resembling the human receptor site to the virus $\mathrm{RBD}$, and the estimation of electromechanical deformation of the cell membrane by $\mathrm{S} 2$ during the prefusion process were included. Possible prevention of viral infection is suggested.
\end{abstract}

Keywords: coronavirus; COVID-19; SARS-CoV-2; fusion; amino acids; electric field; charge; electrostatic potential; anions; vitamin $\mathrm{C}$

\section{Introduction}

Although the total structural charge of SARS-Cov-2 is positive, the structural proteins of SARS-Cov-2 are carrying varied total electric charge related to the amino acid content. The envelope, membrane and nucleocapsid proteins are positive, and the surface spike protein $\mathrm{S}$ is negative. The viral spike protein $S$ is an essential component in the process of SARS-Cov-2 entering the cell. It contains 99 positively, and 111 negatively, charged amino acids. Protein S consists with the two subunits $\mathrm{S} 1$ and $\mathrm{S} 2$. The S1 unit (aa 14-685) contains the receptor binding domain (aa 333-526), RBD, interplaying with the human angiotensin-converting enzyme 2, ACE2, during virus binding to the cell. 
Enzymatic cleavage in the area aa 682-685 in fully reveals subunit S2. Some segments of the S2 unit (aa 686-1273) play an important role in the fusion of SARS-CoV-2 preceding the underlying infection of cells. Although the exact location of the segments, enabling initial lipid membrane boarding can be disputed, the investigation in the conformational conserved area of so called fusion peptide (FP) [1], and in its close proximity seems to be rational. Similar regions to SARS-CoV Extended Bipartite Fusion Platform [2], consisting with 38 amino acids perturbing membrane order in the two neighboring regions, FP1 and FP2, may be also indicated, but they follow the FP (aa 788-806) of SARS-CoV-2 towards C-term.

\section{Observations}

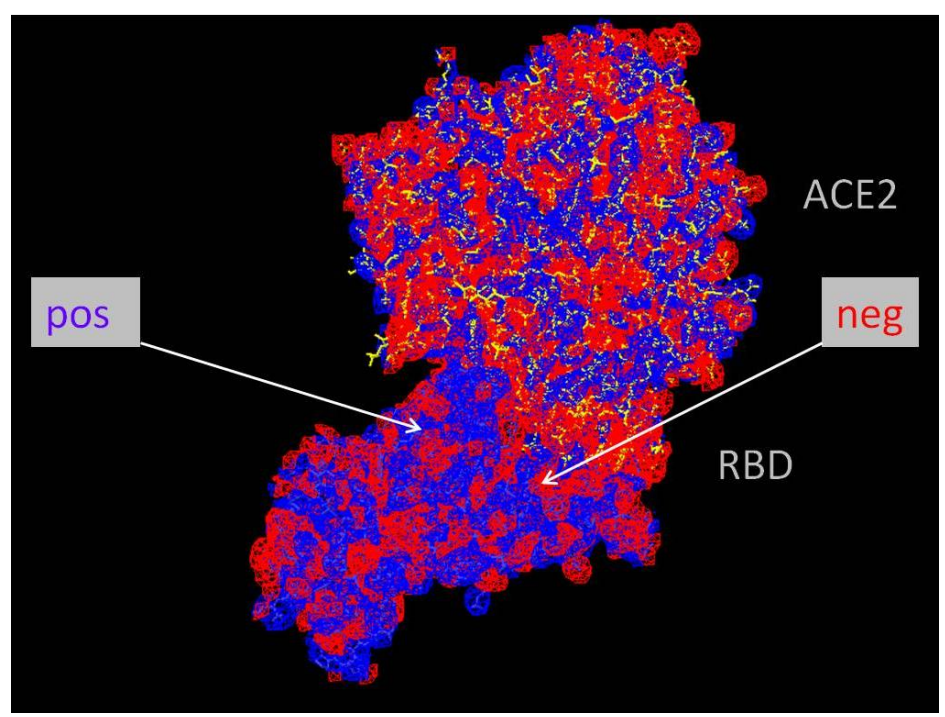

Figure 1. The electrostatic potential (Poisson-Boltzmann method) at the interface of SARS-CoV-2 spike receptor-binding domain (RBD) and human angiotensin-converting enzyme 2 (ACE2). Crystal structure 6M0J was analyzed. The meaning of color area: red-negative, and violet-positive, electric potential. Yellow and blue lines represent ACE2, and RBD backbone. The examples of possible border regions which may interact via Coulomb forces are indicated by arrows. They represent positive Lys and negative Glu at the RBD gate, producing electric dipole, tangential to the RBD-ACE2 interface plane. Computed with Swiss-PdbViewer v4.1.0.

When to consider the interface of SARS-CoV-2 spike receptor-binding domain, RBD, and human angiotensin-converting enzyme 2, ACE2, the inhomogeneity of the electric charge can be seen (Figure 1). The total charged amino acid unbalance in RBD equals 7 positive residues, and in ACE2 there are unbalanced 28 negative residues [3], both contributing to the occurrence of the resultant mezoscopic Coulomb forces between the spike and the receptor. In the stricter border area there is an excess of negative charge with some isolated areas of positive charge, both in RBD and ACE2. In general positive charge prefers protein interior. 


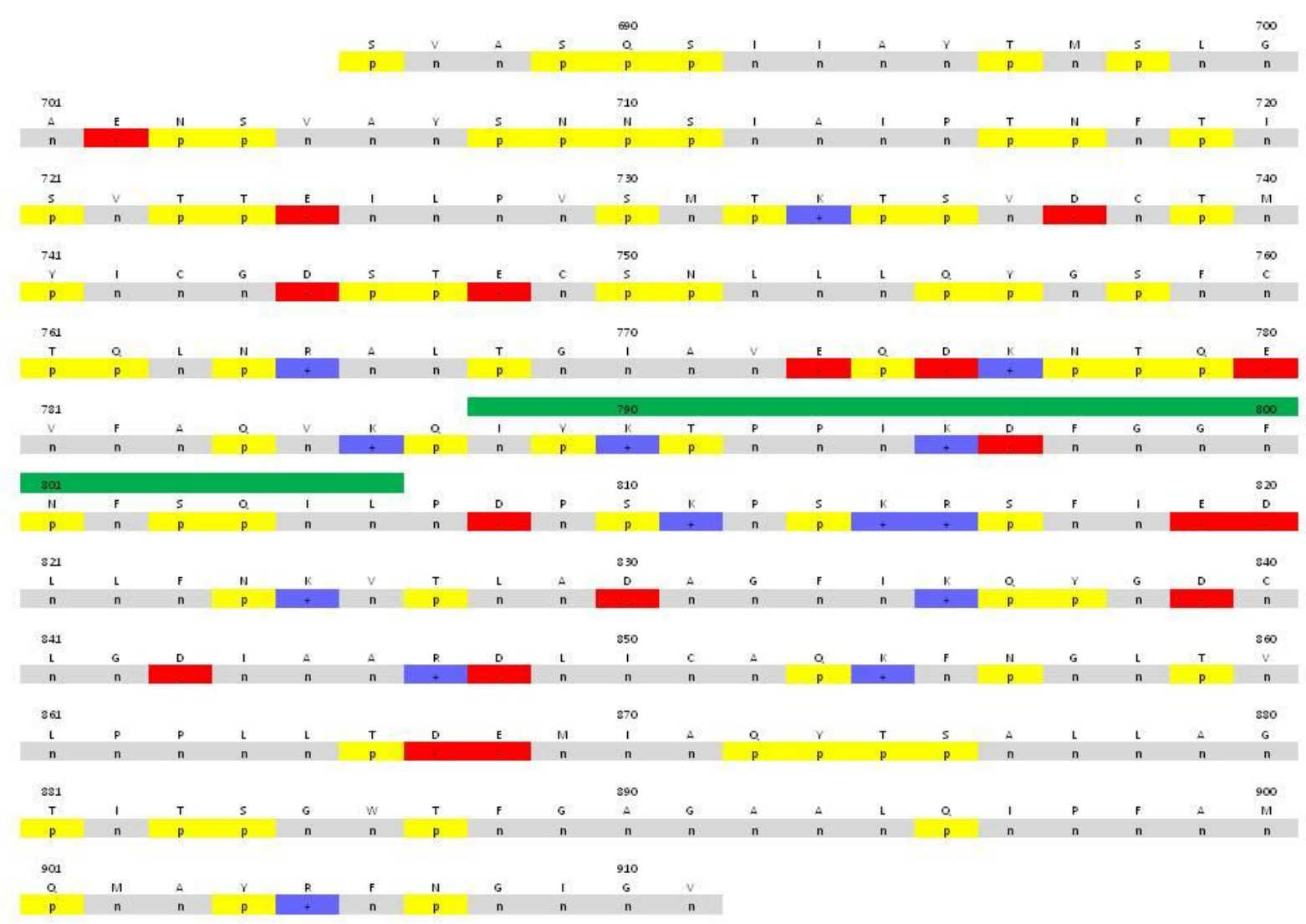

Figure 2. The S2 region (aa 686-911) comprising FP (aa 788-806) of SARS-CoV-2. Color meaning: green-fusion peptide area, blue-positively charged aa, red-negatively charged aa, yellow-polar aa, gray-non-polar aa.

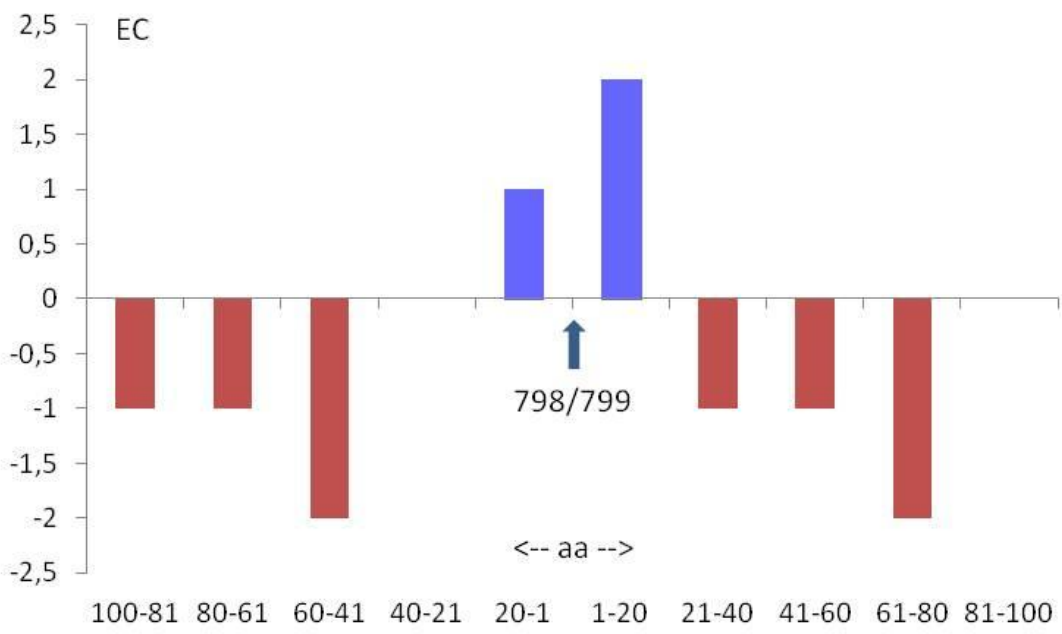

Figure 3. The total electric charge (EC) of charged amino acids in the subsequent areas, 20 amino acids each, on both sides of the position 798/799, which is the center of the considered region of S2 (aa 686-911). The center of comprised FP (aa 788-806) of SARS-CoV-2 falls into the position 797. 
When analyzing the whole region of S2 (aa 686-911) comprising FP, i.e., following the cleavage site (S1/S2) and preceding the heptad repeats regions (HR1 and HR2), it is easy to conclude that charged amino acids (14 positive and 18 negative) are located preferably in the wide central part of discussed aa sequence (Figure 2), with some small bias toward C-term and the clear vanishing at the both strand ends. The positive charge is more condensed $\left(811^{+}-41\right)$ than negative $\left(796^{+}-49\right)$ and prevails in the central area of considered region of S2 (Figure 3).

\section{The idea}

In a case I, considering the possible inhibition of the virus-receptor binding, in the region of the RBD-ACE2 docking site, the cationic ligands should be preferred, or the peptides with the specific charge distribution. Nevertheless, a precise binding of the RBD with the ligand also require the other types of physical interactions, mainly hydrogen bonding, dominating the total binding energy.

\section{Cytosol Extracellular}

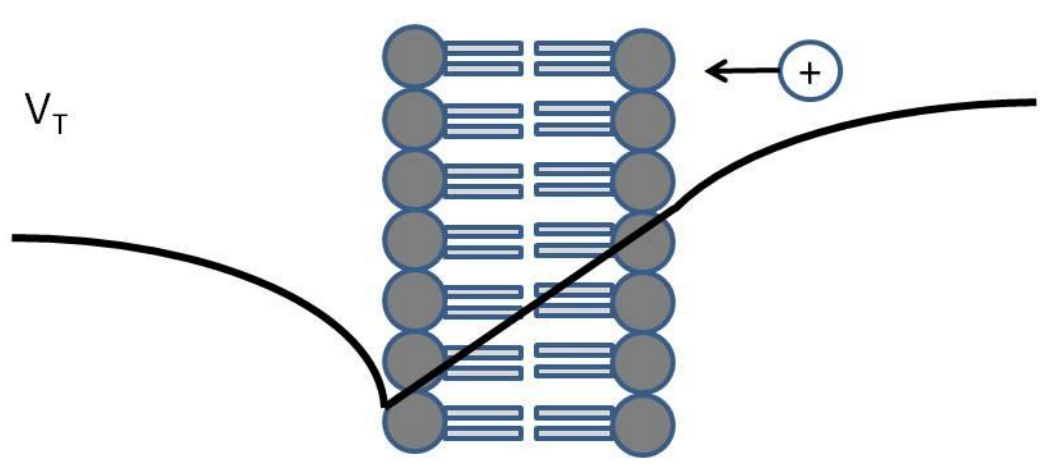

Figure 4. The qualitative predictions of the transmembrane potential VT with the total summary model, Goldman/Hodgkin/Katz- Donnan/Nernst-Ohki.

In a case II, considering the possible inhibition of prefusion, the negative charge of amino acid residues may be in pairs associated with extracellular $\mathrm{Ca}^{2+}$ cations in the vicinity of the cell membrane surface [4], and thus partially neutralized. Amino acids with not compensated positive charge, located relatively closer to the cell membrane, can be electrostatically attracted towards extracellular membrane leaflet, and finally mechanically compress the cell membrane, changing the phospholipids order. The electric field near the extracellular surface driving positive charges towards the cell surface (Figure 4) is predicted by the total summary model, Goldman/Hodgkin/KatzDonnan/Nernst-Ohki [5]. Thus, inhibiting small anionic ligands for FP should be preferred. 


\section{Results}

Case I. An anionic peptide pep ="EEQAKTFLDKFNHEAEDLFYQSS" (net charge -4), inspired by the predicted picture of ACE2 (22-44) binding to virus RBD [6], was analyzed. The method of blind protein-peptide docking (in the vicinity of all target cavities) through a hierarchical algorithm, with an ensemble of peptide conformations generated by the special algorithm, was applied (web server http://huanglab.phys.hust.edu.cn/hpepdock). The obtained docking energy pep-RBD of SARS-CoV-2 is -179 (Figure 5a, HPEPDOCK, [7]). For comparison, the docking energy pep-RBD of MERS-CoV is -199 . The central modification of considered peptide with negatively charged amino acid, namely, the E/N decreases the docking energy score from -179 to -185 (Figure $5 b$, HPEPDOCK, [7]). Following substitution E/K in $5^{\text {th }}$ position decreases the docking energy score to -204 . Stronger binding $(-221)$ can be obtained with the prior residue charge separation, the positive residues at the N-end, and the negative residues at the C-end (KKQATFLFNHALFYQSSEEDEED), respectively, answering in the opposition to the RBD dipole-like charge inhomogeneity (see Fig.1). Following anionic substitution $\mathrm{E} / \mathrm{L}$ in the central $12^{\text {th }}$ position decreases the docking energy score to -230 . The same length anionic peptide, $23 \mathrm{E}$, increases the docking energy up to -81 , and the cationic peptide, $23 \mathrm{~K}$, to -140 , manifesting the importance of the role of the surface negative charge and short-range interactions when approaching the peptide to the RBD. Shorter peptides (10K, 10E) exhibit similar docking energies $(-149,-84)$. The dependence of the docking energy, $\mathrm{E}$, on the number of lysine mers, $\mathrm{n}$, in the polypeptide chain $\mathrm{nK}$, was analyzed in any RBD point and in the RBD-ACE2 docking site (Figure 6), revealing plateau above $\mathrm{N}>8$. Further investigation of the modifications of arbitrary chosen $10 \mathrm{~K}$ peptide shows that leaving the central charged amino acids, KK, substitution the others by the biggest amphiphatic amino acid, tryptophan, and adding the border charged amino acids, $\mathrm{K}$ and E, i.e. (KWWWWKKWWWWE), produce the more effective binding -283 , with the best 10 attempts localized in the close vicinity of RBD-ACE2 docking area (Figure 7).
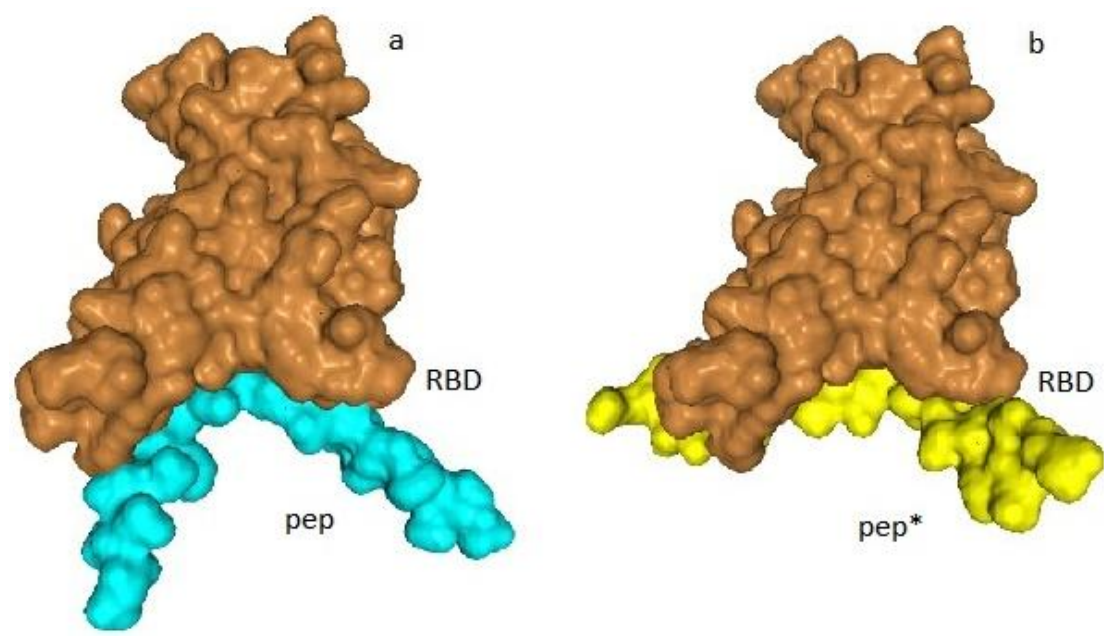

Figure 5. Docking of the peptide pep (a) and modified pep* (b) to RBD (part of the crystal structure 6M0J was analyzed). The pep = "EEQAKTFLDKFNHEAEDLFYQSS". An anionic modification, the substitution $\mathrm{E} / \mathrm{N}$, decreases the docking energy score from -179 , to -185 . The HPEPDOCK algorithm [7] was applied using the hierarchical flexible peptide docking (conformer generation and ensemble docking). 


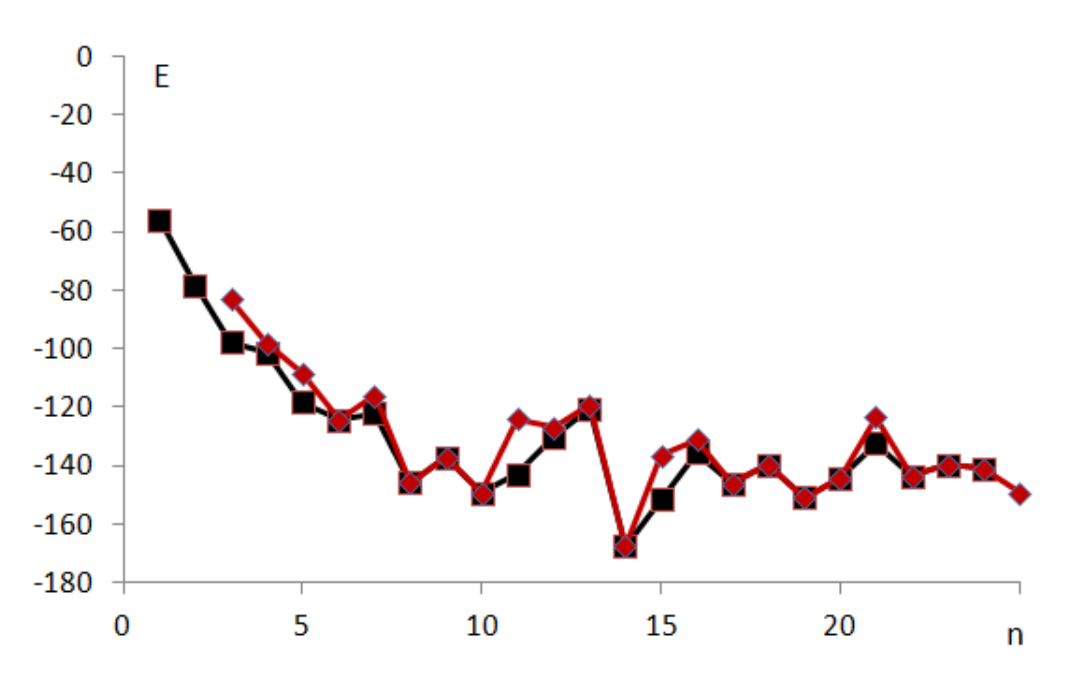

Figure 6. The docking energy score, E (HPEPDOCK algorithm), vs. the number of lysine mers, $\mathrm{n}$, in the polypeptide chain $\mathrm{nK}$. Color meaning: black-maximal docking energy in any RBD point, brown- maximal docking energy in RBD-ACE2 docking site.

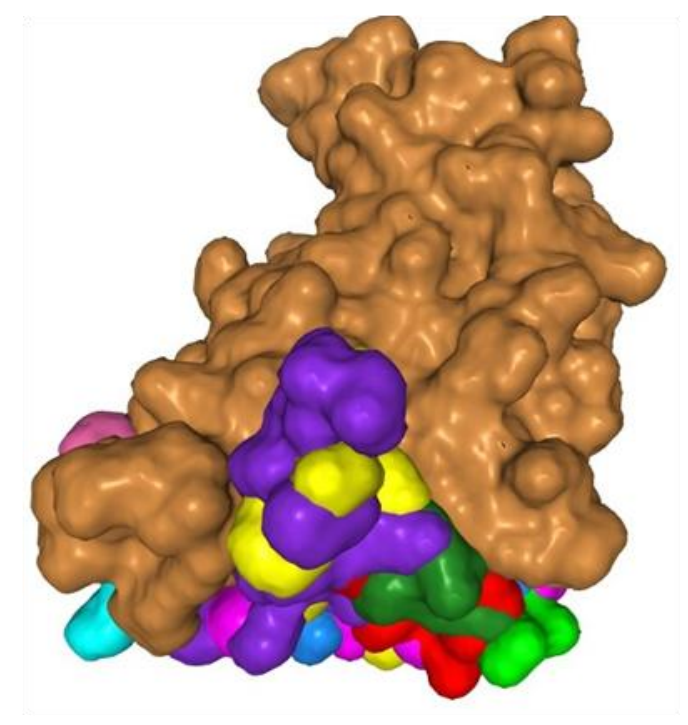

Figure 7. The best energy score docking found for the modified peptide pep* binding to the SARS-CoV-2 RBD. The pep* = "KWWWWKKWWWWE ". RBD was analyzed as a part of the crystal structure 6M0J. A cationic and amphiphatic modification of the initial peptide pep, decreases the docking energy score from -179 , to -283 (HPEPDOCK). Colors indicate 10 best attempts.

Case II. A rough estimation of relative compression of the membrane thickness due to the electric force acting per single positive residue

$$
\mathrm{dh} / \mathrm{h}=-\mathrm{e}^{*} \mathrm{U} /\left(\mathrm{r}^{2} \mathrm{~K}_{\mathrm{A}}\right)
$$


where $\mathrm{e}=1.6^{*} 10^{-19} \mathrm{C}$ is the elementary charge, $\mathrm{U}=0.07 \mathrm{~V}$ is the transmembrane potential, $\mathrm{r}=5^{*} 10^{-10}$ $\mathrm{m}$ is the amino acid effective radius, $\mathrm{K}_{\mathrm{A}}=0.25 \mathrm{~N} / \mathrm{m}$ is the membrane area compressibility modulus, gives the value $\mathrm{dh} / \mathrm{h}=-0.057$.

This for the membrane of the thickness $\mathrm{h}=10 \mathrm{~nm}$ gives the deformational displacement around $\mathrm{dh}=-6$ Angstroms .

\section{Discussion}

In the case I, the long-range electrostatic Coulomb attraction may govern an initial approaching of the SARS-CoV-2 spike S receptor-binding domain, RBD, and human angiotensin-converting enzyme 2, ACE2 (Figure 1). The importance of the electric charge is somewhat confirmed by the evolution trend to conserve the total charge $\mathrm{q}$ in the central region of RBD. For the change relative to SARS-CoV (2003) (aa 323-502), $\Delta \mathrm{q}=0$, at observed mutations in positions of the 12/34 charged amino acids [3]. The total charge change, relative to the central part (181 aa) of MERRS-CoV (6WAR), $\Delta \mathrm{q}=1($ Table 1$)$.

Table 1. The electric charge in the central region of RBD.

\begin{tabular}{llll}
\hline Charged aa & MERS-CoV & SARS-CoV & SARS-CoV-2 \\
$\mathrm{R}(+)$ & 4 & 8 & 9 \\
$\mathrm{~K}(+)$ & 8 & 10 & 8 \\
$\mathrm{D}(-)$ & 7 & 12 & 9 \\
$\mathrm{E}(-)$ & 4 & 4 & 6 \\
Total charged aa & 23 & 34 & 32 \\
Total charge q & +1 & +2 & +2 \\
\hline
\end{tabular}

R- arginine, L -lysine, D - aspartic acid, G -glycine.

It shows that the total charge in the central region of RBD can sometimes be more important than the special content of the charged amino acids. However, a smaller charge of the central region of MERRS-CoV RBD and stronger anionic pep bonding comparing to SARS-CoV-2 indicates the leading role of the local interactions (bridges) in the virus binding, leaving electrostatic forces responsible for the virus-cell approaching phase.

Despite the spike and the receptor charge in the total are negative, the local multipolar (monopolar and dipolar) interactions may produce both attraction and repulsion of approaching proteins. The final results of a peptide pep docking to RBD (see Results) show that precise binding with the receptor also requires the other types of physical interactions in the peptide-protein interface, mainly hydrogen bonding with polar or amphiphatic amino acids. What should be underlined, at the RBD surface cationic peptides could be more binding than anionic ones, due to the prevalence of negative surface charge at the RBD. The length of eight mers seems to be the lower limit without significantly affecting 
the level of the docking energy (Figure 6). The distribution of charge in the peptide is also essential. It may govern the relative orientation of RBD and peptide during approximation, probably by the interaction preferring anti-parallel dipoles (Figure 1). Maximal spacial separation of charge can decrease binding energy $28 \%$. Some small anionic replacements can be energetically favorable, but cationic amino acids in the center of docking peptide seems to be more promising (Figure 7). The best docked peptide "KWWWWKKWWWWE", examined by the FASTA procedure of similarity search (https://www.ebi.ac.uk/Tools/sss/fasta/), does not show significant similarity ( $<55 \%$ identities) to the sequence of any of the known human proteins. The docking energy score -283 is better than that for the best insect peptide of the length 23 aa (-224 [8]).

In the case II, according to the presented estimation of cation pressure on the cell membrane during prefussion, the respective increase in the local membrane area may reach about $6 \%$. It is a serious change. The maximum fractional area change in the RBC cells of the order $3 \%$ may produce lysis [9]. Initial anchor in a few viral positive residues, of a such intensity in the deformation of a phospholipid billayer, may be one of the effective mechanisms of the pre-fusion stabilization of the virus binding at the cell surface and the successive dysfunction of the membrane integrity.

The interactions of single and multiple charged objects of spherical and cylindrical shapes with the surface of charged lipid membranes are in the focus of the relevant recent studies, considering the DNA condensation and coil-globule transition mediated by the deformed membrane [10]. Moreover, the theoretical model of the orientational response of anisotropic rods deposited onto a surface of a soft, elastic substrate was formulated [11]. Virus-mucus interactions in the micrometers layer covering the cells are also important. They can modify the virus electrostatic attraction to the cell surface, e.g. by changing the dielectric constant [12] of the extracellular medium. Non-Gaussian aspect of the diffusion of pathogens in the non-heterogenous network have also been studied in the recent analysis of the bead interactions with mucin gels at different $\mathrm{pH}$ [13].

Regarding Sars-Cov-2 prevention, in a case I, usage well designed peptides resembling ACE2 docking site or cationic polypeptides seem to be reasonable way. In a case II, anions seem to be more effective.

Also recently reported, a new virus receptor, neutropilin-1 [14], facilitates virus entry due to the interaction with the positive (net charge +3) cleavage region S1/S2 (681-PRRSR/SAV-688) [15] of the spike protein, which could be blocked by anions. On the other hand, also there is the expectation of that the cationic peptides $[16,17]$ may bind to cellular receptors involved in viral infection and create an 'antiviral shield' at mucosal surfaces, preventing replication and spread of the coronavirus. Nevertheless, a possible adverse effects should first be examined [18]. The anion gapis usually a positive difference between the concentrations of cations and anions in serum, plasma, or urine. It may be fruitful to check, if the violation of this stabilization by anionic agents decreasing anion gap, e.g., HCO3- NO3-, CO32-, or ascorbate anion (SARS-CoV, Figure 8), could decrease the efficiency of viral fusion, offering some advantages in COVID-19 patients' treatment. It is worth to notice, that anionic water spray in the prophylaxis and treatment of bronchitis was already reported more than six decades ago [19]. A valuable, inspiring input may also come from the industry press release, indicating to the key role of the negative hydroxyl radicals in the process of the virus inactivation, offering by the plasmacluster ions [20] or photocatalytic air cleaning [21] technology. 


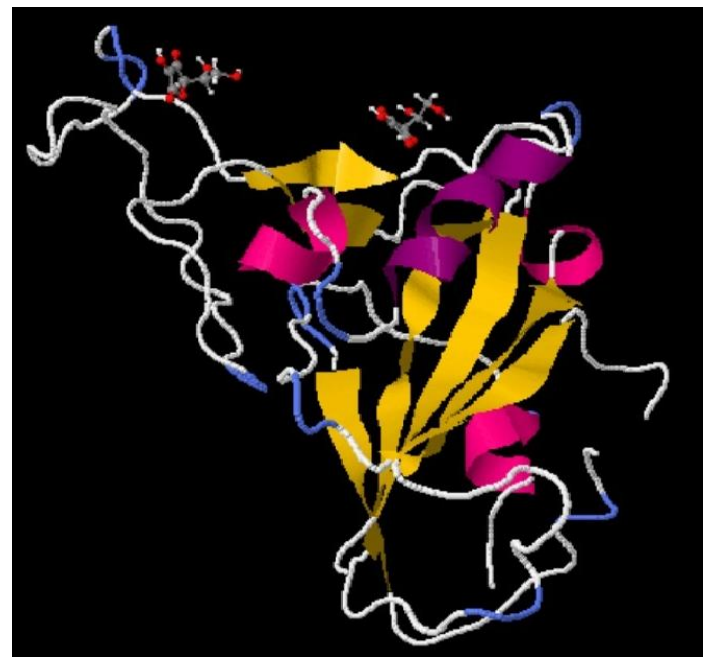

Figure 8. Vitamin C docked to RBD according to SwissDock. Cluster 9/45, fullfitness -821.12 $\mathrm{kcal} / \mathrm{mol}$ (upper right) and cluster 20/45, fullfitness $-820.41 \mathrm{kcal} / \mathrm{mol}$ (upper left). SwissDock is based on the docking algorithm consisting of the following steps: 1 . generation of many binding modes. 2. estimation of energies on a grid. 3. evaluation of energetically favorable modes and clustering. 4. visualization of the most favorable clusters. SwissDock target SARS-CoV (3BGF) and ligand ZINC100006770 were analyzed.

\section{Conclusions}

Presented idea of the electrostatic influence needs further verification as offering a possible basis for the extra care of patients infected by SARS-CoV-2.

\section{Conflict of interest}

The author declares no conflict of interest.

\section{References}

1. Xia S, Liu M, Wang C, et al. (2020) Inhibition of SARS-CoV-2 (previously 2019-nCoV) infection by a highly potent pan-coronavirus fusion inhibitor targeting its spike protein that harbors a high capacity to mediate membrane fusion. Cell Res 30: 343-355.

2. Millet JK, Whittaker GR (2018) Physiological and molecular triggers for SARS-CoV membrane fusion and entry into host cells. Virology 517: 3-8.

3. Lan J, Ge J, Yu J, et al. (2020) Structure of the SARS-CoV-2 spike receptor-binding domain bound to the ACE2 receptor. Nature 581: 215-220.

4. Lai AL, Millet JK, Daniel S, et al. (2017) The SARS-CoV fusion peptide forms an extended bipartite fusion platform that perturbs membrane order in a calcium-dependent manner. $J$ Mol Biol 429: 3875-3892.

5. Benarroch JM, Asally M (2020) The microbiologist's guide to membrane potential dynamics. Trends Microbiol 28: 304-314. 
6. Yang J, Petitjean S, Derclaye S, et al. (2020) Molecular interaction and inhibition of SARS-CoV-2 binding to the ACE2 receptor. Nat Commun: 4541

7. Zhou P, Jin B, Li H, et al. (2018) HPEPDOCK: a web server for blind peptide-protein docking based on a hierarchical algorithm. Nucleic Acids Res 46: W443-W450.

8. Wong FC, Ong JH, Chai TT (2020) Identification of putative cell-entry-inhibitory peptides against SARS-CoV-2 from edible insects: an in silico study. EFood 1: 357-368.

9. Evans EA, Waugh R, Melnik L (1976) Elastic area compressibility modulus of red cell membrane. Biophys J 16: 585.

10. Cherstvy AG, Petrov EP (2014) Modeling DNA condensation on freestanding cationic lipid membranes. Phys Chem Chem Phys 16: 2020-2037.

11. Kumari S, Ye F, Podgornik R (2020) Ordering of adsorbed rigid rods mediated by the Boussinesq interaction on a soft substrate. J Chem Phys 153: 144905.

12. Lonappan A (2012) Novel method of detecting H1N1 using microwaves. J Biomed Sci Eng 5: 476-479.

13. Cherstvy AG, Thapa S, Wagner CE, et al. (2019) Non-Gaussian, non-ergodic, and non-Fickian diffusion of tracers in mucin hydrogels. Soft Matter 15: 2526-2551.

14. Cantuti-Castelvetri L, Ojha R, Pedro LD, et al. (2020) Neuropilin-1 facilitates SARS-CoV-2 cell entry and provides a possible pathway into the central nervous system. BioRxiv preprint https://doi.org/10.1101/2020.06.07.137802.

15. Xia S, Lan Q, Su S, et al. (2020) The role of furin cleavage site in SARS-CoV-2 spike protein-mediated membrane fusion in the presence or absence of trypsin. Signal Transduct TarTher 5: 1-3.

16. Findlay EG, Currie SM, Davidson DJ (2013) Cationic host defence peptides: potential as antiviral therapeutics. Biodrugs 27: 479-493.

17. Boas LCPV, Campos ML, Berlanda RLA, et al. (2019) Antiviral peptides as promising therapeutic drugs. Cell Mol Life Sci 76: 3525-3542.

18. Straus MR, Tang T, Lai AL, et al. (2020) $\mathrm{Ca}^{2+}$ ions promote fusion of Middle East Respiratory Syndrome coronavirus with host cells and increase infectivity. $J$ Virol 94: e00426-20.

19. Franke K (1954) Anionic water spray in the prophylaxis and treatment of bronchitis. Arch Phys Ther 1954 6: 130-132.

20. Susuki T, Kobayashi N (2004) Plasmacluster ions inactivate an airborne corona virus: A world first verification research conducted jointly with the Kitasato Institute. Sharp Company, Press release 2004.

21. Goswami DY (2003) Decontamination of ventilation systems using photocatalytic air cleaning technology. J Sol Energy Eng 125: 359-365.

(C) 2021 the Author(s), licensee AIMS Press. This is an open access AIMS AIMS Press article distributed under the terms of the Creative Commons Attribution License (http://creativecommons.org/licenses/by/4.0) 